\section{Asociaciones entre factores de riesgos psicosociales, dimensiones organizacionales y problemas de salud mental, relacionados con la violencia laboral, en trabajadores de tres hospitales chilenos de alta complejidad}

\author{
Associations between psychosocial risk factors, \\ organizational dimensions, and mental health \\ problems related to workplace violence among \\ workers of three Chilean hospitals of \\ high complexity
}

Associação entre fatores de riscos psicossociais, dimensões organizacionais e problemas de saúde mental relacionados com a violência no trabalho em trabalhadores de três hospitais chilenos de alta complexidade

\section{Resumen}

El objetivo fue analizar la asociación entre factores de riesgos psicosociales, dimensiones organizacionales, violencia laboral e indicadores de problemas de salud mental en tres hospitales chilenos. Mil veintitrés trabajadores respondieron una encuesta, se realizó análisis descriptivos, bivariados (chi-cuadrado) y multivariados (regresión logística con odds ratio como medida de asociación). El 14,7\% de los encuestados reporta violencia laboral. Tienen más oportunidad de reportar violencia laboral los/as trabajadores/as expuestos a ISOSTRAIN $(O R=2,32, p=0,001)$; desequilibrio esfuerzo-recompensa $(O R=5,52$, $p \leq 0,001)$; liderazgo tiránico $(O R=3,02, p \leq 0,001)$ y vulnerabilidad $(O R=1,97, p=0,010)$, respecto a los no expuestos. Los expuestos a ISOSTRAIN $(O R=1,78, p=0,024)$; desequilibrio esfuerzo-recompensa $(O R=2,49$, $p=0,001)$; violencia laboral $(O R=2,13, p=0,003) y$ vulnerabilidad $(O R=2,29, p=0,001)$ tienen más oportunidad de reportar distrés elevado, en relación a los no expuestos. Los expuestos a desequilibrio esfuerzo-recompensa $(O R=2,84, p \leq 0,001)$ y violencia laboral $(O R=1,76, p=0,041)$ tienen más oportunidad de reportar sintomas depresivos que los no expuestos. Los expuestos a desequilibrio esfuerzo-recompensa $(O R=2,07, p \leq 0,001)$ y vulnerabilidad laboral $(O R=1,96, p=0,001)$ tienen más oportunidad de consumir psicotrópicos. Los contextos laborales con factores de riesgos psicosociales, dimensiones organizacionales adversas y vulnerabilidad laboral se asocian a una mayor oportunidad de violencia laboral. Asimismo, trabajadores expuestos a violencia laboral, riesgos psicosociales y vulnerabilidad tienen mayor oportunidad de reportar problemas de salud mental.

Violencia Laboral; Salud Mental; Salud Ocupacional; Síntomas Psíquicos
Andrea Palma-Contreras 1,2

Elisa Ansoleaga 3

doi: 10.1590/0102-311X00084219

\author{
Correspondencia \\ A. Palma-Contreras \\ Doctorado en Psicología, Universidad Diego Portales. \\ Vergara 275, Santiago - 8320000, Chile. \\ anpalma2004@yahoo.es \\ 1 Doctorado en Psicología, Universidad Diego Portales, \\ Santiago, Chile. \\ 2 Facultad de Psicología, Universidad San Sebastián, \\ Santiago, Chile. \\ 3 Facultad de Psicología, Universidad Diego Portales, \\ Santiago, Chile.
}




\section{Introducción}

La violencia laboral ha sido considerada la epidemia del siglo XXI, generando efectos en la salud mental de los trabajadores 1,2, y contribuye a las tasas ausentismo laboral en nuestro país 3 . En Chile, el trabajo en el sector salud ha sido identificado como uno de los ámbitos de mayor riesgo de exposición a violencia laboral 4 , sometiendo a los trabajadores a demandas laborales emocionalmente complejas.

La violencia laboral es considerada en este artículo desde el concepto de intimidación en el lugar de trabajo 5 (p. 671), esto es, "la exposición directa a un comportamiento negativo sistemático y prolongado en el trabajo" proveniente de superiores, compañeros de trabajo, subalternos y usuarios. La violencia laboral ha sido identificada como uno de los mayores estresores en el trabajo, debido a su prevalencia y a los dañinos efectos en las personas y las organizaciones 6.

Con respecto a variables asociadas con la violencia laboral, la evidencia indica que dentro de ellas se encuentran la exposición a factores de riesgos psicosociales laborales y a determinadas dimensiones organizacionales 7,8. Los riesgos psicosociales laborales son definidos como "aquellas características de las condiciones de trabajo y, sobre todo, de su organización que afectan la salud de las personas a través de mecanismos psicológicos y fisiológicos" 9. Así, existirían formas de organizar el trabajo sanitario que son riesgosas para la salud de los trabajadores: altas exigencias psicológicas, escasas posibilidades de trabajo activo, bajo apoyo social y el desequilibrio esfuerzo-recompensa ${ }^{3}$, entre otros, los que a la vez aumentan la posibilidad de experimentar conflictos con otros trabajadores y con usuarios pudiendo derivar en situaciones de violencia laboral.

Respecto a las dimensiones organizacionales, la literatura identifica que ciertas características de la cultura organizacional y del liderazgo pueden influir en el desarrollo de la violencia laboral. La cultura organizacional es el "conjunto de creencias y valores compartidos por los miembros de una misma organización (que) influye en sus comportamientos” 10 (p. 288). En este artículo se estudian tres dimensiones culturales que han sido evidenciadas como promotoras de la violencia laboral 7: cultura organizacional estricta (versus laxa), centrada en el trabajo (versus el trabajador) y orientada a los resultados (versus proceso). Por último, en línea con investigación previa, se considera que el liderazgo destructivo 11,12, específicamente el estilo de liderazgo laissez-faire 13 y el liderazgo tiránico 11 promueven la violencia laboral. El primero de ellos porque se trata de líderes que no ejercen sus funciones, y en el segundo estilo, porque están dispuestos a utilizar las amenazas e intimidación para lograr que los subalternos cumplan las metas.

Por otra parte, la literatura ha mostrado de manera consistente que la exposición a violencia laboral es un fenómeno que se asocia con síntomas y problemas de salud mental tales 14,15,16,17 como el distrés, la sintomatología depresiva y el consumo de psicotrópicos.

A pesar de la alta relevancia del fenómeno de la violencia laboral a nivel mundial, la evidencia en nuestro país es insuficiente para movilizar a la acción en la materia. Por ello, la relevancia de esta investigación radica en que permitirá conocer las dimensiones organizacionales y factores de riesgos psicosociales que promueven la violencia laboral, así como también su papel en generar problemas de salud mental en un caso poco estudiado y de alta prevalencia, como lo es, el ámbito del trabajo en salud en Chile.

En este artículo se analizan variables asociadas con la violencia laboral en tres hospitales chilenos de alta complejidad. Se hipotetiza que la exposición a factores de riesgos psicosociales laborales y dimensiones organizacionales adversas incrementan la posibilidad de reportar violencia laboral (H1), mientras que la exposición a violencia laboral, factores de riesgos psicosociales y dimensiones organizacionales adversas aumentan la oportunidad de que los trabajadores presenten indicadores de problemas de salud mental (H2).

En síntesis, el propósito de este artículo es analizar la asociación entre factores de riesgos psicosociales, dimensiones organizacionales (liderazgo y cultura organizacional), presencia de violencia laboral e indicadores de problemas de salud mental (distrés, sintomatología depresiva, consumo de psicotrópicos), en trabajadores de tres hospitales chilenos de alta complejidad. 


\section{Métodos}

\section{Diseño y procedimientos}

Se realizó un estudio empírico analítico, cuantitativo, no experimental, y transversal. Desde mayo a noviembre de 2018, se recolectó información con un cuestionario autoadministrado en papel, de autorreporte, aplicado a 1.023 funcionarios (302 hombres y 721 mujeres) de tres hospitales chilenos de alta complejidad, correspondiente al 10,5\% de la dotación total de dichos hospitales (Tabla 1).

En los tres hospitales el muestreo se realizó segmentando por sexo y estamento. En uno el muestreo fue aleatorio dentro de los segmentos (muestreo estratificado); en los otros dos -por marco muestral inaccesible- se encuestó a funcionarios disponibles para contestar dentro de cada segmento (muestreo por cuotas).

El estudio recibió la aprobación de los comités de ética de la Universidad Diego Portales (no 0282017) y de los tres hospitales (NO2886). Los participantes firmaron un consentimiento informado, resguardando confidencialidad y voluntariedad.

\section{Variables e instrumentos}

\section{- Violencia laboral}

La violencia laboral puede ser de tipo personal, laboral o físico 18. Se midió con el Negative Act Questionnaire-Revised, validado en Chile por González ${ }^{19}$. Se trata de una escala Likert de 22 ítems con 5 alternativas de respuesta (Nunca, Alguna vez, $\mathrm{Al}$ menos una vez al mes, Al menos una vez a la semana, A diario). Se consideró víctimas de violencia laboral a quiénes informan dos o más comportamientos negativos al menos una vez a la semana durante un período de 6 meses 20 .

Tabla 1

Descripción de la muestra. Estudio desarrollado en tres hospitales de alta complejidad de Chile, 2018.

\begin{tabular}{lccc}
\hline & $\begin{array}{c}\text { Mujeres } \\
\mathbf{n}(\%)\end{array}$ & $\begin{array}{c}\text { Hombres } \\
\mathbf{n}(\%)\end{array}$ & $\begin{array}{c}\text { Total } \\
\mathbf{n}(\%)\end{array}$ \\
\hline $\begin{array}{l}\text { Total } \\
\text { Rangos de edad (años) }\end{array}$ & $721(70,5)$ & $302(29,5)$ & $1.023(100,0)$ \\
$18-30$ & $206(29,1)$ & & \\
$31-40$ & $193(27,3)$ & $74(24,7)$ & $280(27,8)$ \\
$41-50$ & $152(21,5)$ & $90(30,0)$ & $214(28,1)$ \\
$51-60$ & $126(17,8)$ & $62(20,7)$ & $176(17,5)$ \\
61 y más & $31(4,4)$ & $50(16,7)$ & $55(5,5)$ \\
Estamentos & & $24(8,0)$ & $101(9,9)$ \\
Administrativos & $81(11,3)$ & & $127(12,4)$ \\
Auxiliares & $59(8,2)$ & $20(6,6)$ & $343(33,6)$ \\
Técnicos & $292(40,6)$ & $68(22,6)$ & $204(20,0)$ \\
Personal médico & $93(12,9)$ & $51(16,9)$ & $246(24,1)$ \\
Otros profesionales no médicos & $195(27,1)$ & $111(36,9)$ & \\
Tipo de contrato & & $51(16,9)$ & $294(28,9)$ \\
Titular (indefinido) & $211(29,4)$ & $83(27,7)$ & $488(47,9)$ \\
Contrata (plazo fijo anual) & $336(46,8)$ & $152(50,7)$ & $81(8,0)$ \\
Honorarios & $64(8,9)$ & $17(5,7)$ & $155(15,2)$ \\
Otro & $107(14,9)$ & $48(16,0)$ & \\
\hline
\end{tabular}




\section{- Riesgos psicosociales laborales}

(i) Demandas psicológicas: incluye demandas cuantitativas y demandas emocionales. Las demandas cuantitativas se refieren al modo de trabajar y a las restricciones de la organización para completar las tareas (velocidad, cantidad de trabajo, tiempo para las tareas, recepción de demandas contradictorias, trabajo mentalmente exigente) 21. Las demandas emocionales 22 consisten en mantenerse emocionalmente distante, ocultamiento de emociones y opiniones 23. Se midieron con el Job Content Questionnaire (JCQ) de Karasek et al. 21, validado por Ansoleaga et al. 22, y la Escala de Demandas Emocionales (Instrumento SUSESO-ISTAS 21) 23. Escala Likert de nueve ítems con cuatro categorías de respuesta desde "muy en desacuerdo" (1) a "muy de acuerdo" (4). Los puntajes oscilaron entre 9 y 36 , y se dicotomizó con punto de corte: mediana = 23 puntos.

(ii) Esfuerzo extrínseco: compuesto por el número y ritmo de tareas y las interrupciones durante el trabajo 24. Se midió con el Effort-Reward Imbalance Questionnaire (ERI) 24. Escala Likert compuesta por tres ítems del cuestionario ERI, con cuatro categorías de respuesta desde "muy en desacuerdo" (1) a "muy de acuerdo" (4). Los puntajes oscilaron entre 3 y 12, el punto de corte fue la mediana $=8$.

(iii) Recompensas: estima y respeto, seguridad, promoción y salario 24 . Se midieron con el ERI 24. Escala Likert compuesta por siete ítems del cuestionario ERI, con cuatro categorías de respuesta desde "muy en desacuerdo" (1) a "muy de acuerdo" (4). Los puntajes oscilaron entre 7 y 28 , el punto de corte fue la mediana $=17$.

(iv) Desequilibrio esfuerzo-recompensas: falta de reciprocidad entre los esfuerzos realizados en el trabajo y las recompensas. Se midió con el ERI 24. Escala Likert compuesta por tres ítems de esfuerzo y siete de recompensa con cuatro categorías de respuesta desde "muy en desacuerdo" (1) a "muy de acuerdo" (4). Se consideró con desequilibrio esfuerzo-recompensa los puntajes superiores a 1 en la siguiente fórmula:

$\Sigma$ puntaje esfuerzo/ $\Sigma$ puntaje recompensas x $0,4285^{\prime}$

donde, 0,4285 es producto de la división de 3/7, correspondiente a los tres ítems de esfuerzo y los siete de recompensa 24 .

(v) Latitud decisional: se refiere al control (posibilidad de decidir cómo hacer el trabajo e influencia sobre su trabajo) y al uso de habilidades (posibilidad de aprender cosas nuevas y nivel de habilidades requeridas para el trabajo) 21 . Se midió con el JCQ de Karasek et al. 21, versión validada en Chile por Ansoleaga et al. 22. Escala Likert de cinco ítems (tres de habilidades y dos de control), cuatro alternativas de respuesta con cuatro categorías de respuesta desde "muy en desacuerdo" (1) a "muy de acuerdo" (4). Los puntajes oscilan entre 5 y 20, se dicotomizó con punto de corte: mediana $=15$ puntos.

(vi) Soporte social: se refiere al apoyo instrumental y emocional de compañeros y jefatura 18 . Se midió con el JCQ de Karasek et al. 21, versión validada en Chile por Ansoleaga et al. 22. Los puntajes oscilan entre 6 y 24, el punto de corte: mediana $=18$.

(vii) ISOSTRAIN: presencia simultánea de altas demandas psicológicas, baja latitud decisional (tensión psíquica) y bajo soporte social 25. Escala Likert de diez ítems con cuatro alternativas de respuesta desde "muy en desacuerdo" (1) a "muy de acuerdo" (4).

\section{- Dimensiones organizacionales}

(i) Cultura organizacional: de acuerdo al modelo de Hofstede 26, se distinguió entre: (a) Cultura organizacional con control estricto vs. laxo: grado de formalidad dentro de la organización; (b) Cultura organizacional orientada al trabajo $v s$. a los trabajadores: responsabilidad sólo por el desempeño laboral vs. responsabilidad respecto a su bienestar; y (c) Cultura organizacional orientada al proceso vs. a los resultados: centradas en rutinas técnicas y burocráticas centradas en el logro.

Se midió con un instrumento elaborado en el marco del Proyecto FONDECYT Regular no 1170239, en función de las tres dimensiones del modelo de Hofstede 26. Es un diferencial semántico con seis categorías de respuesta para cada dimensión. Se dicotomizó con punto de corte: mediana = 3 . (ii) Liderazgo: se distinguió entre: (a) Liderazgo tiránico: exposición a líderes con comportamientos que apoyan el logro de objetivos de la organización, pero obtienen resultados a expensas del bienestar 
de los subordinados 11 y (b) Liderazgo laissez-faire: liderazgos sin conducción, que no cumplen las responsabilidades ni deberes asignados 11 .

Se midió con la escala de liderazgo destructivo 12 y la escala de liderazgo laissez-faire 13, validadas en Chile por González ${ }^{19}$. El instrumento consistió en una escala Likert de cuatro ítems para liderazgo tiránico y tres para laissez-faire con cuatro categorías de respuesta desde "nunca" (0) a "casi siempre" (3). Las respuestas para ambos tipos de liderazgo se dicotomizaron, considerando expuestos a quienes contestaron "bastante a menudo" o "casi siempre" a alguna pregunta de cada escala.

\section{- Salud mental}

(i) Distrés psicológico: malestar psicológico, compuesto por síntomas de: depresión, inquietud, fatiga, sentimiento de culpa y ansiedad 27. Se midió con la escala de distrés psicológico de Kessler (K6) 27. Escala Likert de seis ítems con cinco alternativas de respuesta, desde "nunca" (0) a "siempre" (4). Se dicotomizó la variable, punto de corte $=0>7$ (distrés elevado o muy elevado).

(ii) Sintomatología depresiva: presencia conjunta de estado de ánimo depresivo y pérdida de interés o placer para la mayoría de las actividades habituales 28 . Se midió con la mini escala de síntomas depresivos, compuesta por dos ítems (anhedonia y distimia) 28. Se trata de dos preguntas con respuesta dicotómica (sí/no) para anhedonia y distimia. Se considera presencia de sintomatología depresiva en quienes contestaron positivamente ambos ítems.

(iii) Consumo de psicotrópicos: prevalencia año de consumo de ansiolíticos, antidepresivos o hipnóticos 28 , testeada en Chile 29. Fue evaluado con la pregunta: "Durante los últimos 12 meses, ha tomado de manera regular, todos los días o algunas veces por semana, medicamentos para: a) ¿disminuir la ansiedad o el nerviosismo?; b) ¿ayudar a dormir? c) ¿remontar el ánimo tales como antidepresivos?” Se consideró presencia de consumo de psicotrópicos en quiénes contestaron positivamente al menos uno de los tres ítems.

\section{- Variables de control}

(i) Vulnerabilidad laboral: definida como "temores que influyen en cómo los trabajadores perciben su propia capacidad para interactuar con empleadores y supervisores” 30 (p. 181). Se midió con una escala Likert de siete ítems con cuatro alternativas de respuesta (desde "muy en desacuerdo" a "muy de acuerdo") 31. Se consideró con vulnerabilidad a quiénes contestaron "positivamente" a tres o más preguntas.

(ii) Estrechez económica: percepción de dificultades económica a fin de mes 32 . Se consultó con la pregunta: “...pensando en el total de ingresos mensuales de su hogar, este... a) Les alcanza bien, pudiendo ahorrar; b) Les alcanza justo, sin grandes dificultades; c) No les alcanza, tienen algunas dificultades; d) No les alcanza, tienen grandes dificultades". Se dicotomizó respecto a quiénes presentan dificultades económicas a fin de mes (c y d) y quiénes no las presentan (a y b).

(iii) Suceso vital estresante: muerte de algún familiar; accidente laboral o común y/o haber sido víctima de una situación violenta los últimos seis meses 32 . Pregunta con respuesta dicotómica (sí/no) para cada suceso. Se dicotomizó respecto a quiénes presentan al menos un suceso estresante.

(iv) Precariedad contractual: hace referencia a contratos con escasa seguridad laboral 28. Se consideró precarios los contratos a "honorarios" y "otros" y no precarios los contratos "planta" y "contrata". (v) Baja estabilidad laboral: percepción de baja estabilidad laboral. Se midió con un ítem del ERI $24:$ "mi estabilidad laboral es baja". Se consideró con baja estabilidad a quienes contestaron "muy de acuerdo" o "de acuerdo".

(vi) Remuneración fija o variable: autorreporte de remuneración (fija/variable) 29. Se midió mediante un ítem con respuesta categórica de cuatro alternativas: (a) fija; (b) tiene una parte fija y una variable; (c) es variable en su totalidad; (d) no sabe. Se dicotomizó considerando fija (a) y variable (b y c).

(vii) Sexo: autorreporte. Se midió con una pregunta de respuesta categórica: hombre-mujer.

(viii) Edad: años de vida. 


\section{Análisis estadísticos}

Se realizaron análisis con estadística descriptiva y bivariada (pruebas de chi-cuadrado). Luego, se estimaron modelos multivariados de regresión logística para predecir (a) la oportunidad de ocurrencia de la violencia laboral, en función de factores de riesgos psicosociales, dimensiones organizacionales y variables control; y (b) problemas de salud mental en función de violencia laboral y de todas las variables mencionadas; se utilizó odds ratio (OR) como medida de asociación (intervalo del 95\% de confianza -IC95\%), porque lo que interesa es comparar grupos expuestos y no expuestos en función de un factor. En los modelos se incluyeron todas las variables independientes expuestas en el apartado anterior que no implicaban colinealidad. Los datos se analizaron conjuntamente para los tres hospitales, a solicitud del Comité de Ética de uno de ellos. Se mantuvo en los modelos de regresión logística las variables que resultaban teóricamente relevantes y que en las pruebas bivariadas resultaron estadísticamente significativas en su asociación con violencia laboral y/o al menos dos de los tres problemas de salud mental estudiados. Los análisis se realizaron con SPSS Statistics 22 (https://www. ibm.com/). Cabe señalar que los resultados bivariados pueden ser solicitados a las autoras.

\section{Resultados}

Como se aprecia en la Tabla 2, 14,7\% de los encuestados reportan violencia laboral, sin existir diferencias significativas entre hombres y mujeres $(\mathrm{p}=0,863)$. A su vez, una proporción relevante de encuestados reportó exposición a factores de riesgos psicosociales: $61,2 \%$ bajo soporte social, 56,1\% desequilibrio esfuerzo-recompensa y el 18,6\% de la muestra reportó ISOSTRAIN.

En relación a las dimensiones organizacionales cabe destacar que un 83,5\% reportó una cultura organizacional orientada al trabajo, el 14,9\% señaló estar expuesto a liderazgo tiránico y el 27,9\% a liderazgo laissez-faire.

Respecto a la salud mental, más de un quinto de los encuestados consume algún psicotrópico (21,9\%), 17,2\% presenta distrés elevado o muy elevado y $15 \%$ sintomatología depresiva. Los factores de riesgos psicosociales y los problemas de salud mental son mayores entre mujeres: reportan menor latitud decisional, mayores esfuerzos y menores recompensas que los hombres $(\mathrm{p}<0,05)$, así como mayor consumo de psicotrópicos $(\mathrm{p}<0,01)$.

$\mathrm{Al}$ analizar por estamento, la mayoría de quienes reportan exposición a violencia laboral son técnicos $(34,7 \%)$ o profesionales no médicos (22,9\%). Coincidentemente, la mayoría de los que reportan distrés elevado pertenecen al estamento técnico $(40,7 \%)$ o profesionales no médicos $(22,1 \%)$.

La Tabla 3 presenta los resultados de un modelo de regresión logística, cuya variable de resultado es la violencia laboral, mientras que las variables independientes son: factores de riesgos psicosociales, dimensiones organizacionales y variables control -sexo, edad, vulnerabilidad, contrato precario, baja estabilidad laboral, remuneración variable, estrechez económica y exposición a suceso vital estresante-. Los expuestos a ISOSTRAIN tienen una oportunidad dos veces mayor de reportar violencia laboral $(\mathrm{OR}=2,32, \mathrm{p}=0,001)$, comparado con quienes no están expuestos a isostran, los que reportan desequilibrio esfuerzo-recompensa también tienen mayor oportunidad de reportar violencia laboral $(\mathrm{OR}=5,52, \mathrm{p} \leq 0,001)$ versus los que no reportan desequilibrio esfuerzo-recompensa y hubo asociaciones significativas de aquellos que reportan exposición a liderazgo tiránico $(O R=3,02, p \leq 0,001)$ y de quienes reportan vulnerabilidad $(\mathrm{OR}=1,97, \mathrm{p}=0,010)$ con violencia laboral, en comparación con quienes no reportan exposición a liderazgo tiránico ni vulnerabilidad.

La Tabla 4 presenta los resultados del modelo de regresión logística, cuyas variables de resultado son los problemas de salud mental y las variables independientes fueron: factores de riesgos psicosociales, dimensiones organizacionales, violencia laboral y variables de control -sexo, edad, vulnerabilidad, contrato precario, baja estabilidad laboral, remuneración variable, estrechez económica y exposición a suceso vital estresante-. Los que reportaron exposición a violencia laboral tienen mayor oportunidad de reportar distrés elevado $(\mathrm{OR}=2,13, \mathrm{p}=0,003)$ y sintomatología depresiva $(\mathrm{OR}=1,76$, $\mathrm{p}=0,041)$ que los no expuestos a violencia laboral. No se encontraron asociaciones significativas de violencia laboral con consumo de psicotrópicos ( $\mathrm{p}>0,05)$. 
Tabla 2

Prevalencia de variables principales. Estudio desarrollado en tres hospitales de alta complejidad de Chile, 2018.

\begin{tabular}{|c|c|c|c|c|}
\hline Variable & $\begin{array}{c}\text { Hombres } \\
\text { n (\%) }\end{array}$ & $\begin{array}{c}\text { Mujeres } \\
\text { n (\%) }\end{array}$ & $\begin{array}{l}\text { Valor de } p \\
\qquad\left(\chi^{2}\right)\end{array}$ & $\begin{array}{l}\text { Total } \\
\text { n (\%) }\end{array}$ \\
\hline Violencia laboral & $43(15,0)$ & $101(14,6)$ & 0,863 & $144(14,7)$ \\
\hline \multicolumn{5}{|l|}{ Riesgos psicosociales } \\
\hline Altas demandas psicológicas & $134(45,3)$ & $360(51,6)$ & 0,066 & $494(49,7)$ \\
\hline Baja latitud decisional & $90(30,2)$ & $268(37,9)$ & 0,021 & $358(35,6)$ \\
\hline JOBSTRAIN & $44(15,0)$ & $136(19,7)$ & 0,076 & $180(18,3)$ \\
\hline Bajo soporte social & $186(61,6)$ & $436(61,0)$ & 0,855 & $622(61,2)$ \\
\hline ISOSTRAIN & $38(12,9)$ & $114(16,6)$ & 0,146 & $152(18,6)$ \\
\hline Altos esfuerzos & $113(37,7)$ & $320(44,8)$ & 0,037 & $433(42,7)$ \\
\hline Bajas recompensas & $137(46,4)$ & $378(54,2)$ & 0,025 & $515(51,9)$ \\
\hline Desequilibrio esfuerzo-recompensa & $150(51,5)$ & $398(58,1)$ & 0,059 & $548(56,1)$ \\
\hline \multicolumn{5}{|l|}{ Dimensiones organizacionales } \\
\hline Cultura organizacional orientada al trabajo & $237(79,8)$ & $597(85,0)$ & 0,041 & $834(83,5)$ \\
\hline Cultura organizacional con control estricto & $95(32,6)$ & $247(35,4)$ & 0,400 & $342(34,6)$ \\
\hline Exposición a liderazgo tiránico & $43(14,4)$ & $107(15,0)$ & 0,060 & $150(14,9)$ \\
\hline Exposición a liderazgo laissez-faire & $90(30,2)$ & $189(27,0)$ & 0,296 & $279(27,9)$ \\
\hline \multicolumn{5}{|l|}{ Variables de control } \\
\hline Suceso vital estresante & $83(27,5)$ & $196(27,2)$ & 0,922 & $279(27,3)$ \\
\hline Estrechez económica & $59(19,6)$ & $176(24,6)$ & 0,085 & $235(23,1)$ \\
\hline Vulnerabilidad laboral & $111(37,0)$ & $304(42,6)$ & 0,099 & $415(40,9)$ \\
\hline Precariedad contractual & $65(21,7)$ & $171(23,2)$ & 0,459 & $236(23,2)$ \\
\hline Remuneración variable & $125(41,9)$ & $333(46,3)$ & 0,209 & $458(45,0)$ \\
\hline Baja estabilidad laboral & $66(21,9)$ & $209(29,2)$ & 0,017 & $275(27,1)$ \\
\hline \multicolumn{5}{|l|}{ Problemas de salud mental } \\
\hline Distrés psicológico elevado & $45(15,2)$ & $127(18,1)$ & 0,265 & $172(17,2)$ \\
\hline Sintomatología depresiva & $39(13,0)$ & $114(15,9)$ & 0,249 & $153(15,0)$ \\
\hline Consumo de psicotrópicos & $47(16,0)$ & $168(24,3)$ & 0,004 & $215(21,9)$ \\
\hline
\end{tabular}

Finalmente, factores de riesgos psicosociales también se asociaron con la violencia laboral. Quienes reportaron ISOSTRAIN ( $\mathrm{OR}=1,78, \mathrm{p}=0,024)$, desequilibrio esfuerzo-recompensa $(\mathrm{OR}=2,49$, $\mathrm{p}=0,001)$, vulnerabilidad $(\mathrm{OR}=2,29, \mathrm{p}=0,001)$, remuneración variable $(\mathrm{OR}=1,76, \mathrm{p}=0,007) \mathrm{y}$ estrechez económica $(\mathrm{OR}=2,08, \mathrm{p}=0,001)$ presentaron una mayor oportunidad de reportar distrés elevado versus quienes no informan de dichos factores. Quienes reportaron desequilibrio esfuerzorecompensa $(\mathrm{OR}=2,84, \mathrm{p} \leq 0,001)$, remuneración variable $(\mathrm{OR}=1,72, \mathrm{p}=0,012)$ y estrechez económica $(\mathrm{OR}=2,32, \mathrm{p} \leq 0,001)$ tienen mayor oportunidad de reportar síntomas depresivos versus quienes no reportan dichas condiciones y aquellos que reportaron desequilibrio esfuerzo-recompensa $(\mathrm{OR}=2,07, \mathrm{p} \leq 0,001)$ y vulnerabilidad $(\mathrm{OR}=1,96, \mathrm{p}=0,001)$, tienen mayor oportunidad de consumo de psicotrópicos en comparación con quiénes no los reportan. 
Tabla 3

Asociaciones de riesgos psicosociales y dimensiones organizacionales con violencia laboral en trabajadores de tres hospitales chilenos de alta complejidad, 2018.

\begin{tabular}{|c|c|c|c|}
\hline & \multicolumn{3}{|c|}{ Violencia laboral } \\
\hline & OR & Valor de $p$ & IC95\% \\
\hline \multicolumn{4}{|l|}{ Riesgos psicosociales } \\
\hline ISOSTRAIN & 2,32 & 0,001 & $1,40-3,89$ \\
\hline Desequilibrio esfuerzo-recompensa & 5,52 & $<0,001$ & $2,86-10,67$ \\
\hline \multicolumn{4}{|l|}{ Dimensiones organizacionales } \\
\hline Cultura organizacional orientada al trabajo & 1,97 & 0,133 & $0,81-4,77$ \\
\hline Cultura organizacional con control estricto & 0,60 & 0,055 & $0,36-1,01$ \\
\hline Exposición a liderazgo tiránico & 3,02 & $<0,001$ & $1,81-5,04$ \\
\hline Exposición a liderazgo laissez-faire & 1,39 & 0,174 & $0,87-2,24$ \\
\hline \multicolumn{4}{|l|}{ Variables de control } \\
\hline Vulnerabilidad laboral & 1,97 & 0,010 & $1,18-3,30$ \\
\hline Precariedad contractual & 1,76 & 0,057 & $0,98-3,14$ \\
\hline Baja estabilidad laboral & 1,10 & 0,727 & $0,66-1,82$ \\
\hline Remuneración variable & 0,68 & 0,090 & 0,43-1,06 \\
\hline Estrechez económica & 1,09 & 0,752 & $0,66-1,79$ \\
\hline Suceso vital estresante & 1,53 & 0,074 & $0,96-2,44$ \\
\hline
\end{tabular}

IC95\%: intervalo del 95\% de confianza; OR: odds ratio.

Tabla 4

Asociación entre riesgos psicosociales, dimensiones organizacionales y violencia laboral con problemas de salud mental en trabajadores de tres hospitales chilenos de alta complejidad, 2018.

\begin{tabular}{|c|c|c|c|c|c|c|c|c|c|}
\hline & \multicolumn{3}{|c|}{ Distrés psicológico elevado } & \multicolumn{3}{|c|}{ Sintomatología depresiva } & \multicolumn{3}{|c|}{ Consumo de psicotrópicos } \\
\hline & OR & Valor de p & IC95\% & OR & Valor de p & IC95\% & OR & Valor de p & IC95\% \\
\hline \multicolumn{10}{|l|}{ Riesgos psicosociales } \\
\hline ISOSTRAIN & 1,78 & 0,024 & $1,08-2,93$ & 0,90 & 0,710 & $0,52-1,57$ & 1,13 & 0,617 & $0,70-1,84$ \\
\hline $\begin{array}{l}\text { Desequilibrio esfuerzo- } \\
\text { recompensa }\end{array}$ & 2,49 & 0,001 & $1,47-4,22$ & 2,84 & $<0,001$ & $1,66-4,85$ & 2,07 & $<0,001$ & $1,38-3,10$ \\
\hline \multirow{2}{*}{\multicolumn{10}{|c|}{$\begin{array}{l}\text { Dimensiones } \\
\text { organizacionales }\end{array}$}} \\
\hline & & & & & & & & & \\
\hline $\begin{array}{l}\text { Cultura organizacional } \\
\text { orientada al trabajo }\end{array}$ & 1,32 & 0,443 & $0,65-2,70$ & 0,88 & 0,705 & $0,46-1,69$ & 1,36 & 0,288 & $0,77-2,40$ \\
\hline $\begin{array}{l}\text { Cultura organizacional } \\
\text { con control estricto }\end{array}$ & 0,91 & 0,691 & $0,58-1,44$ & 1,02 & 0,928 & $0,64-1,62$ & 0,72 & 0,098 & $0,49-1,06$ \\
\hline $\begin{array}{l}\text { Exposición a liderazgo } \\
\text { tiránico }\end{array}$ & 1,51 & 0,117 & $0,90-2,53$ & 1,53 & 0,123 & $0,89-2,63$ & 0,95 & 0,842 & $0,58-1,57$ \\
\hline $\begin{array}{l}\text { Exposición a liderazgo } \\
\text { laissez-faire }\end{array}$ & 1,11 & 0,657 & $0,70-1,76$ & 0,78 & 0,321 & $0,48-1,28$ & 1,29 & 0,207 & $0,87-1,93$ \\
\hline \multicolumn{10}{|l|}{ Variables de control } \\
\hline Vulnerabilidad laboral & 2,29 & 0,001 & $1,43-3,68$ & 1,40 & 0,182 & $0,86-2,26$ & 1,96 & 0,001 & $1,31-2,93$ \\
\hline Precariedad contractual & 0,83 & 0,489 & $0,48-1,42$ & 0,54 & 0,032 & $0,30-0,95$ & 0,63 & 0,072 & $0,38-1,04$ \\
\hline Baja estabilidad laboral & 0,94 & 0,800 & $0,58-1,53$ & 0,84 & 0,508 & $0,51-1,40$ & 0,59 & 0,025 & $0,38-0,94$ \\
\hline Remuneración variable & 1,76 & 0,007 & $1,16-2,65$ & 1,72 & 0,012 & $1,13-2,64$ & 0,93 & 0,693 & $0,65-1,33$ \\
\hline Estrechez económica & 2,08 & 0,001 & $1,34-3,24$ & 2,32 & $<0,001$ & $1,47-3,65$ & 1,15 & 0,513 & $0,76-1,75$ \\
\hline Suceso vital estresante & 1,66 & 0,021 & $1,08-2,54$ & 2,44 & $<0,001$ & $1,59-3,76$ & 1,76 & 0,003 & $1,21-2,57$ \\
\hline Violencia laboral & 2,13 & 0,003 & $1,29-3,52$ & 1,76 & 0,041 & $1,02-3,01$ & 0,78 & 0,338 & $0,47-1,30$ \\
\hline
\end{tabular}

IC95\%: intervalo del 95\% de confianza; OR: odds ratio. 


\section{Discusión}

El objetivo de este artículo es analizar la asociación entre factores de riesgos psicosociales, dimensiones organizacionales, violencia laboral e indicadores de problemas de salud mental en tres hospitales chilenos de alta complejidad. Se encontró asociación de: ISOSTRAIN, desequilibrio esfuerzorecompensa y liderazgo tiránico con violencia laboral; violencia laboral, ISOSTRAIN y desequilibrio esfuerzo-recompensa con distrés elevado; desequilibrio esfuerzo-recompensa con síntomas depresivos y con consumo de psicotrópicos.

Utilizando la definición de Mikkelsen \& Einarsen 20, se encontró un reporte de violencia laboral del 14,7\%, por el 10\% reportado por trabajadores dependientes de diversas ocupaciones de tres áreas metropolitanas de Chile 19. El mayor reporte de violencia laboral en funcionarios sanitarios concuerda con reportes previos que indican que el sector salud es uno de los más afectados 4 , esto se puede vincular con su trabajo que es especialmente demandante debido a: trabajo por turnos, alta demanda emocional y la estructura burocrática en que se desarrolla 33. Internacionalmente, en estudios que han utilizado un criterio similar al de este estudio, se ha encontrado prevalencias de violencia laboral: entre 2 y $8 \% 16 ; 6,2 \% 34 ; 19 \% 35$.

Respecto a las variables organizacionales, este estudio reporta un $18,6 \%$ de presencia de ISOSTRAIN, frente a lo encontrado en estudios que indican un $10 \%$ en población general $36 ; 12,4 \%$ en trabajadores del Servicio Médico Legal de Chile 37 y 15\% en un hospital del alta complejidad de Santiago 38. La mayor presencia de ISOSTRAIN que en muestras de personal sanitario de estudios anteriores 37,38 puede vincularse con el avance en la implementación de prácticas que tienen como base el paradigma del new public management ${ }^{39}$, cuya instalación en el sistema sanitario impacta en: el modelo de atención, la gestión de recursos humanos, los sistemas de control, el uso de indicadores, los procesos de evaluación de desempeño que se vinculan al establecimiento de metas cuantitativas, entre otras 32 , lo que se puede traducir en la exposición a factores de riesgos psicosociales.

Continuando con variables organizacionales el desequilibrio esfuerzo-recompensa $(56,1 \%)$ reportado en esta muestra es inferior a dos de los estudios mencionados, donde se obtuvo 68,7\% 37 y $67 \% 38$. La menor presencia de desequilibrio esfuerzo-recompensa que en muestras de personal sanitario de estudios anteriores puede vincularse con la distribución de los participantes según estamento, en particular, el porcentaje de personal médico participante en esta muestra (20\%) es superior a la de los dos estudios con los que se compara (15\% y 11\% respectivamente), tratándose del estamento con menores prevalencias de exposición, debido a que tienen jornadas laborales parciales 40 , más poder organizativo, mejor remunerado y más reconocimiento social por su trabajo.

No existen estudios previos publicados que analicen elementos de cultura organizacional con el modelo de Hofstede en trabajadores sanitarios. Tampoco existe evidencia publicada en Chile desde el modelo de liderazgo destructivo. A nivel internacional, un estudio en población trabajadora de Noruega, encontró prevalencias de un 2,4\% para liderazgo tiránico y un 13,1\% para laissez-faire 11, muy por debajo de lo reportado en este estudio (14,9 y 27,9\% respectivamente).

Un 17,2\% reportó distrés elevado; lo que está por debajo de lo encontrado en estudios previos en funcionarios de la salud 37,38 y en población trabajadora general ${ }^{36}$. La sintomatología depresiva reportada está por debajo de investigaciones previas 37,38. Similar situación ocurre con el consumo de psicotrópicos $(21,9 \%)$ siendo inferior al 31\% 37 y el 25\% 38 encontrado antes. Esto puede explicarse por las diferencias en la composición de las muestras entre estudios, levantando la interrogante por las desigualdades sociales presentes en el trabajo en salud que pueden repercutir tanto en las condiciones laborales, como en el resultado de salud de esta población.

Respecto a las variables asociadas con violencia laboral, en coincidencia con la literatura internacional 41,42, se encontró que la presencia de violencia laboral se relaciona con dimensiones de la organización del trabajo, los estilos de liderazgo y las cultura organizacional, que generan un aumento en la presión por eficiencia y competencia 8 . De este modo, la presencia de factores de riesgos psicosociales (como el ISOSTRAIN y el desequilibrio esfuerzo-recompensa), una cultura organizacional orientada al trabajo y la exposición a líderes tiránicos se asocian a una mayor chance de violencia laboral.

Coincidentemente con estudios anteriores 43,44, la sensación de vulnerabilidad, como un indicador de la diferencia de poder entre el perpetrador y la víctima, se expresa en temores -a perder el empleo, ser reemplazado fácilmente, entre otros- que influyen en cómo los trabajadores perciben su capacidad 
para interactuar con sus superiores 30 y aumenta la oportunidad de reportar violencia laboral. Este hallazgo se condice con lo que indica la Organización Internacional del Trabajo (OIT), respecto a que la violencia laboral posee una relación inversamente proporcional con el acceso al trabajo decente y afecta principalmente a trabajadores vulnerables 45 , porque las interacciones en el interior de las organizaciones reproducen las desigualdades y discriminaciones de la sociedad general 43 .

Por otra parte, se observaron asociaciones de violencia laboral con distrés elevado y sintomatología depresiva, no así respecto a consumo de psicotrópicos. Ello, se condice con estudios previos en que se ha encontrado asociación de violencia laboral con depresión 46 y estrés laboral 2 y difiere de estudios como el de Dement et al. 1 que reporta asociación con uso de antidepresivos y ansiolíticos. A su vez, resulta importante destacar que la exposición a factores de riesgos psicosociales y la vulnerabilidad también se asocian positivamente con la oportunidad de experimentar problemas de salud mental, aun sin violencia laboral.

En conclusión, respecto a la primera hipótesis se confirma la asociación significativa entre la exposición a dimensiones organizacionales adversas (ISOSTRAIN, desequilibrio esfuerzo-recompensa, liderazgo tiránico) y el reporte de violencia laboral al 99\% de confianza, controlando por variables relevantes tales como: edad, sexo, vulnerabilidad, estrechez económica, precariedad contractual, entre otras.

Respecto a la segunda hipótesis, se confirma la asociación entre la exposición a factores de riesgo psicosociales (desequilibrio esfuerzo-recompensa, ISOSTRAIN) y la exposición a violencia laboral y el distrés elevado, controlando por variables relevantes como edad, sexo, vulnerabilidad, estrechez económica, remuneración variable, suceso vital estresante, entre otras. En segundo lugar, se confirmó la asociación entre dimensiones organizacionales adversas (desequilibrio esfuerzo-recompensa) y consumo de psicotrópicos, controlando por variables relevantes: edad, sexo, vulnerabilidad, baja estabilidad laboral, suceso vital estresante, entre otras. Cabe explicitar que no se encontró asociación entre exposición a violencia laboral y consumo de psicotrópicos. En tercer lugar, no se encontró asociación entre dimensiones organizacionales adversas propias de la cultura organizacional o del estilo de liderazgo (tiránico o laissez-faire) con sintomatología derivada de problemas de salud mental, controlando por las demás variables.

Entre las limitaciones del estudio se encuentra que su carácter transversal impide reportar causalidad; la deseabilidad social en las respuestas respecto a violencia laboral y sintomatología derivada de problemas de salud mental y la imposibilidad de extrapolar los resultados a la población general por tratarse de una muestra no aleatoria.

Finalmente, atendiendo a que la mayor oportunidad de reportar violencia laboral se asocia con contextos laborales que presentan factores de riesgos psicosociales, dimensiones organizacionales adversas y vulnerabilidad, unido a que la exposición a violencia laboral, riesgos psicosociales y vulnerabilidad en el trabajo se asocia con indicadores de problemas de salud mental de los trabajadores, resulta relevante proponer políticas más efectivas que reduzcan la exposición de los trabajadores sanitarios a condiciones laborales adversas que atiendan a las recomendaciones de la OIT y la Organiazción Mundial de la Salud (OMS) ${ }^{4}$ referentes a: desarrollar cultura organizacional centradas en lo humano, explicitar declaraciones de buen trato, generar conciencia en los actores involucrados, asegurar la suficiente dotación de personal, propiciar estilos de dirección que respeten la dignidad, establecer protocolos para trabajadores con más riesgo de violencia laboral, entre otros.

Dada la distribución de la fuerza de trabajo en salud para futuros estudios, se sugiere realizar análisis exhaustivos relacionados con cada categoría profesional. 


\section{Colaboradores}

A. Palma-Contreras contribuyó con la concepción y diseño del trabajo, recolección/obtención de resultados, análisis e interpretación de datos, redacción del manuscrito y aprobación de su revisión final. E. Ansoleaga contribuyó con la concepción y diseño del trabajo, análisis e interpretación de datos, redacción del manuscrito, revisión crítica del manuscrito y aprobación de su versión final.

\section{Informaciones adicionales}

ORCID: Andrea Palma-Contreras (0000-00026281-1509); Elisa Ansoleaga (0000-0003-01820126).

\section{Referencias}

1. Dement J, Lipscomb H, Schoenfisch A, Pompeii A. Impact of hospital type II violent events: use of psychotropic drugs and mental health services. Am J Ind Med 2014; 57:627-39.

2. Itzhaki M, Peles-Bortz A, Kostisky H, Barnoy D, Filshtinsky V, Bluvstein I. Exposure of mental health nurses to violence associated with job stress, life satisfaction, staff resilience, and post-traumatic growth. Int J Ment Health Nurs $2015 ; 24: 403-12$.

3. Superintendencia de Seguridad Social. Estadísticas de seguridad social. Informe anual 2017. http://www.suseso.gob.cl/607/arti cles-496701_archivo_01.pdf (accedido el 25/ Ene/2019).

4. Organización Internacional del Trabajo; Consejo Internacional de Enfermeras; Organización Mundial de la Salud; Internacional de Servicios Públicos. Directrices marco para afrontar la violencia laboral en el sector de salud. https://www.who.int/violence_injury_ prevention/violence/workplace/es/ (accedido el 15/Dic/2018).

5. Notelaers G, Einarsen S. The world turns at 33 and 45: defining simple cutoff scores for the Negative Acts Questionnaire-Revised in a representative sample. European Journal of Work and Organizational Psychology 2013; 22:67082 .

\section{Agradecimientos}

Este artículo fue realizado en el marco del Proyecto FONDECYT Regular 2017 no 1170239: Prevalencia, Formas de Manifestación y Factores Facilitadores de la Violencia Laboral y sus Efectos en la Salud Mental en Población Asalariada en Chile, Atendiendo Desigualdades Sociales y de Género, el cual cuenta con financiamiento de la Comisión Nacional de Investigación Científica y Tecnológica (CONICYT) y la Beca CONICYT de doctorado nacional no 21170967. Además, se agradece la asesoría metodológica de la Dra. Mónica Gerber Plüss.
6. di Martino V. Relationship between work stress and workplace violence in the health sector. https://www.who.int/violence_injury_ prevention/violence/interpersonal/WVstress paper.pdf (accedido el 26/Ene/2019).

7. Olsen E, Bjaalid G, Mikkelsen A. Work climate and the mediating role of workplace bullying related to job performance, job satisfaction, and work ability: a study among hospital nurses. J Adv Nurs 2017; 73:2709-19.

8. Salin D. Bullying and organisational politics in competitive and rapidly changing work environments. International Journal of Management and Decision Making 2003; 4:35-46.

9. Instituto Sindical de Trabajo, Ambiente y Salud. Manual para la evaluación de riesgos psicosociales en el trabajo. Método ISTAS (CoPsoQ). http://www.istas.ccoo.es/descar gas/m_metodo_istas21.pdf (accedido el 22/ $\mathrm{Feb} / 2019$ ).

10. Ruiz Y, Naranjo J. La investigación sobre cultura organizacional en Colombia: una mirada desde la difusión en revistas científicas. Diversitas Perspectiv Psicol 2012; 8:285-307.

11. Aasland M, Skogstad A, Notelaers G, Nielsen M, Einarsen S. The prevalence of destructive leadership behaviour. British Journal of Management 2010; 21:438-52. 
12. Einarsen S, Skogstad A, Schanke M. The nature, prevalence, and outcomes of destructive leadership: a behavioral and conglomerate approach. In: Schyns B, Hansbbrough T, editors. When leadership goes wrong. Destructive leadership, mistakes and ethical failures. Charlotte: Information Age Publishing; 2010. p. 145-74.

13. Bass B, Avolio B. Transformational leadership development: manual for the multifactor leadership questionnaire. Palo Alto: Consulting Psychologists Press; 1990.

14. Zafar W, Khan UR, Siddiqui SA, Jamali S, Razzak JA. Workplace violence and self-reported psychological health: coping with post-traumatic stress, mental distress, and burnout among physicians working in the emergency departments compared to other specialties in Pakistan. J Emerg Med 2016; 50:167-77.

15. Malik OF, Schat ACH, Shahzad A, Raziq MM, Faiz R. Workplace psychological aggression, job stress, and vigor: a test of longitudinal effects. J Interpers Viol 2018; [Epub ahead of print].

16. Sperduti A, Hindle D, Shessel A, Pidgeon B, Akmal H, Chaulk G, et al. Treating too lightly? Radiation therapists' experiences of workplace violence when providing care to cancer patients and their families. J Med Imaging Radiat Sci 2018; 49:56-61.

17. Zhao S, Xie F, Wang J, Shi Y, Zhang S, Han X, et al. Prevalence of workplace violence against Chinese nurses and its association with mental health: a cross-sectional survey. Arch Psychiatr Nurs 2018; 32:242-7.

18. Einarsen S, Hoel H, Notelaers G. Measuring exposure to bullying and harassment at work: validity, factor structure and psychometric properties of the Negative Acts QuestionnaireRevised. Work Stress 2009; 23:24-44.

19. González A. Propiedades psicométricas de escala de liderazgo destructivo y Cuestionario de Conductas Negativas-Revisado en una muestra multiocupacional de trabajadores dependientes chilenos de Gran Santiago, Gran Valparaíso y Gran Concepción [Dissertación de Maestría]. Santiago: Universidad Diego Portales; 2019.

20. Mikkelsen E, Einarsen S. Bullying in Danish worklife: prevalence and health correlates. European Journal of Work and Organizational Psychology 2001; 10:393-413.

21. Karasek R, Brisson C, Kawakami N, Houtman I, Bongers P, Amick B. The Job Content Questionnaire (JCQ): an instrument for internationally comparative assessments of psychosocial job characteristics. J Occup Health Psychol 1998; 3:322-55.

22. Ansoleaga E, Montaño R, Vézina M. Validation of two complementary instruments for measuring work stress in Chilean workers. Scandinavian Journal of Organizational Psychology 2013; 5:5-14.
23. Superintendencia de Seguridad Social. Manual del método del cuestionario SUSESO/ ISTAS21. Versiones completa y breve. https:// www.suseso.cl/613/articles-481095_archi vo_03.pdf (accedido el 02/Abr/2019).

24. Siegrist J, Wege N, Pühlhofer F, Wahrendorf M. A short generic measure of work stress in the era of globalization: effort-reward imbalance. Int Arch Occup Environ Health 2009; 82:1005-13.

25. Karasek R, Theorell T. Healthy work: stress, productivity, and the reconstruction of working life. New York: Basic Books; 1990.

26. Hofstede G. Dimensionalizing cultures: the Hofstede model in context. Online Psychology and Culture 2011;2:1-26.

27. Kessler RC, Andrews G, Colpe LJ, Hiripi E, Mroczek DK, Normand SL, et al. Short screening scales to monitor population prevalences and trends in non-specific psychological distress. Psychol Med 2002; 32:959-76.

28. Vézina M, Cloutier E, Stock S, Lippel K, Fortin E, Delisle A, et al. Enquête Québécoise sur les conditions de travail, d'emploi, de santé et sécurité du travail (EQCOTESST). https://www.irsst.qc.ca/media/documents/Pu bIRSST/R-691.pdf (accedido el 30/Mar/2018).

29. Centro de Estudios de la Mujer; Universidad de Ottawa. Encuesta Araucaria. Santiago: Centro de Estudios de la Mujer; 2011.

30. Casas L. The effectiveness of sexual harassment law in Chile: from theory to practice [Tesis de Doctorado]. Ottawa: University of Ottawa; 2016.

31. Amable M. La precariedad laboral y su impacto en la salud. Un estudio en trabajadores asalariados en España [Tesis de Doctorado]. Santiago: Universitat Pompeu Fabra; 2008.

32. Ansoleaga E. Sintomatología depresiva y consumo riesgoso de alcohol en trabajadores expuestos a factores psicosociales laborales adversos: un asunto pendiente en salud pública [Tesis de Doctorado]. Santiago: Facultad de Medicina, Universidad de Chile; 2013.

33. Ansoleaga E, Artaza O, Suárez J, editores. Personas que cuidan personas: dimensión humana y trabajo en salud. Santiago: Organización Panamericana de la Salud/Organización Mundial de la Salud; 2012.

34. Nielsen M, Skogstad A, Matthiesen S, Glasøto L, Aasland M, Notelaers G, et al. Prevalence of workplace bullying in Norway: comparisons across time and estimation methods. European Journal of Work and Organizational Psychology 2009; 18:81-101.

35. Giorgi G. Workplace bullying in academia creates a negative work environment: an Italian study. Employee Responsibilities \& Rights Journal 2012; 24:261-75.

36. Ansoleaga E, Vézina M, Montaño R. Síntomas depresivos y distrés laboral en trabajadores chilenos: condiciones diferenciales para hombres y mujeres. Cad Saúde Pública 2014; 30:107-18. 
37. Ansoleaga E, Urra M. Efectos del riesgo psicosocial laboral en la salud mental de funcionarios del Servicio Médico Legal de Chile. Vertex Rev Argent Psiquiatr 2015; 26:427-34.

38. Ansoleaga E. Indicadores de salud mental asociados a riesgo psicosocial laboral en un hospital público. Rev Med Chile 2015; 143:47-55.

39. Hood C. A public management for all season? Public Adm 1991; 69:3-19.

40. Ansoleaga E, Toro JP, Godoy L, Stecher A, Blanch J. Malestar psicofisiológico en profesionales de la salud pública de la Región Metropolitana. Rev Med Chile 2011; 139:118591.

41. An Y, Kang J. Relationship between organizational culture and workplace bullying among Korean nurses. Asian Nurs Res 2016; 10:234-9.

42. Wei-Quan L, Jiang W, Le-Xin Y, Sheng-Chao Z, Meng-Juan J, Hui-Shan Z, et al. Workplace violence and job performance among community healthcare workers in China: the mediator role of quality of life. Int J Environ Res Public Health $2015 ; 12: 14872-86$.
43. Díaz X, Mauro A, Ansoleaga E, Toro JP. Violencia de género en el trabajo en Chile. Un campo de estudio ignorado. Cienc Trab 2017; 19:42-8.

44. Salin D. Ways of explaining workplace bullying: a review of enabling, motivating and precipitating structures and processes in the work environment. Hum Relat 2003; 56:1213-32.

45. Pillinger J. Violence and harassment against women and men in the world of work: trade union perspective and action. http://www. ilo.org/wcmsp5/groups/public/---ed_ dialogue/---actrav/documents/publication/ wcms_546645.pdf (accedido el 27/Feb/2019).

46. Giorgi G, Mikayo A, Arenas A, Shoss M, LeónPérez J. Exploring personal and organizational determinants of workplace bullying and its prevalence in a Japanese sample. Psychol Violence $2013 ; 3: 185-97$. 


\section{Abstract}

The objective was to analyze the association between psychosocial risk factors, organizational dimensions, workplace violence, and indicators of mental health problems in three Chilean hospitals. A total of 1,023 workers answered a questionnaire, and descriptive, bivariate (chi-square), and multivariate (logistic regression with odds ratio as the measure of association) analyses were performed. Of all the interviewees, $14.7 \%$ reported workplace violence. Increased odds of workplace violence were associated with exposure to ISOSTRAIN $(O R=2.32, p=0.001)$, effort-reward imbalance $(O R=5.52, p \leq 0.001)$, tyrannical leadership $(O R=3.02, p \leq 0.001)$, and vulnerability $(O R=1.97, p=0.010)$. Workers exposed to ISOSTRAIN $(O R=1.78, p=0.024)$, effort-reward imbalance $(O R=2.49, p=0.001)$, workplace violence $(O R=2.13, p=0.003)$, and vulnerability $(O R=2.29, p=0.001)$ showed increased odds of high stress when compared to unexposed workers. Workers exposed to effort-reward imbalance $(O R=2.84, p \leq 0.001)$ and workplace violence $(O R=1.76, p=0.041)$ showed higher odds of reporting depressive symptoms. Those exposed to effort-reward imbalance $(O R=2.07, p \leq 0.001)$ and work vulnerability $(O R=1.96, p=0.001)$ showed higher odds of consuming psychoactive drugs. Work contexts with psychosocial risk factors, adverse organizational dimensions, and work vulnerability were associated with higher odds of workplace violence. Workers exposed to workplace violence, psychosocial risks, and vulnerability have higher odds of reporting mental health problems.

Workplace Violence; Mental Health;

Occupational Health; Psychic Symptoms

\section{Resumo}

O objetivo foi analisar a associação entre fatores de riscos psicossociais, dimensões organizacionais, violencia no trabalho e indicadores de problemas de saúde mental em três hospitais chilenos. Mil e vinte e três trabalhadores responderam um questionário, foram realizadas análises descritivas, bivariadas (qui-quadrado) e multivariadas (regressão logística com odds ratio como medida de associação). Relataram violência no trabalho, 14,7\% dos entrevistados. Os/as trabalhadores/as expostos/as a ISOSTRAIN $(O R=2,32, p=0,001)$ têm maior chance de relatar violência no trabalho; desequilíbrio esforço-recompensa $(O R=5,52, p \leq$ $0,001)$; liderança tirânica $(O R=3,02, p \leq 0,001)$ e vulnerabilidade $(O R=1,97, p=0,010)$, em relação aos não expostos. Os expostos a ISOSTRAIN $(O R=1,78, p=0,024)$; desequilíbrio esforço-recompensa $(O R=2,49, p=0,001)$; violência no trabalho $(O R=2,13, p=0,003)$ e vulnerabilidade $(O R=2,29, p=0,001)$ têm uma maior chance de reportar distresse elevado, em relação aos não expostos. Os expostos a desequilíbrio esforço-recompensa $(O R=2,84, p \leq 0,001)$ e violência laboral $(O R=1,76, p=0,041)$ têm maior chance de reportar sintomas depressivos do que os nãos expostos. Os expostos a desequilíbrio esforço-recompensa $(O R=2,07, p \leq 0,001)$ e vulnerabilidade laboral $(O R=1,96, p=0,001)$ têm maior chance de consumir psicotrópicos. Os contextos laborais com fatores de riscos psicossociais, dimensões organizacionais adversas e vulnerabilidade laboral foram associados a uma maior chance de violência no trabalho. Além disso, trabalhadores expostos à violência no trabalho, riscos psicossociais e vulnerabilidade têm maior chance de reportar problemas de saúde mental.

Violência no Trabalho; Saúde Mental; Saúde do Trabalhador; Sintomas Psíquicos
Recibido el 04/May/2019

Versión final presentada el 24/Ago/2019

Aprobado el 16/Sep/2019 